\title{
A new Hybrid Mode-Matching Method for the Analysis of Inductive Obstacles and Discontinuities
}

H. Esteban*, S. Cogollos, A. Vidal, V.E. Boria, and M. Ferrando Depto. de Comunicaciones, Univ. Politècnica de València Camino de Vera $s / n, 46022$ Valencia, SPAIN Tel: +34-96-3877820 Fax: +34-96-3877309

\author{
E-mail: vboria@upvnet.upv.es
}

\section{Abstract}

A new and efficient hybrid mode-matching method for the analysis of arbitrarily shaped inductive obstacles and discontinuities is presented. An open-space spectral method is used to model the electrical
behavior of the inductive problem, and the mode-matching technique is then employed for obtaining a multimode scattering matrix representation. This new method is revealed very useful for the accurate and efficient analysis of inductive problems including very arbitrary geometries. Results for some ciassical inductive problems covered in the literature are shown, thus confirring the accuracy of the results provided
by the new techrique.

I. InTRODUCTION

The inductive obstacles and discontinuities in waveguiding structures has been traditionally a key element of many microwave devices, such as manifold diplexers and multiplexers placed at the payload of communication satellites [1], and microwave filters for communiplaced at the payload of communication satellites $[1]$, and microwave filters for communicontinuities has received a considerable attention in the technical literature. The study of inductive discontinuities have been performed by two groups of techniques. The first one considers only the fundamental mode interactions and is very efficient computationally [3] On the other hand, the second group takes into account higher-mode interactions but is less efficient numerically. In this second group we can find the well known mode-matchin technique [4], a method based on a multimode admittance matrix representation [5] and more recently an efficient integral equation technigue [6]. For the analysis of inductive posts more recently an efficient integral equation technique [6]. For the analysis of inductive posts a classical single-mode method based on an integral equation can be already found in $[7]$ and a multimode technique combinin

In this paper a new and efficient hybrid muitimode technique is described for the analysis In this paper a new and efficient hybrid multimode technique is described for the analysis
of inductive obstacles and discontinuities with generic shapes. This new technique combines properly an open-space spectral method with the well known multimode mode-matching technique. The spectral method is used for the accurate characterization of the inductive problem under consideration, then the mode-matching technique is used to efficiently obtain a multimode scattering matrix representing the problem. Proceeding in this way inductive obstacles of any shape (triangular or square, for instance), generic inductive steps and also inductive bends can be efficiently and accurately analyzed. To validate the new method derived, results for an inductive window and iris and for inductive circular metallic and dielectric posts are offered. Comparisons with available data from the technical literature are also included.

\section{THEORY}

The general layout of the problem is depicted in figure 1

In order to characterize the scattering behavior of the inductive problem (discontinuities and obstacles), an open-space spectral formulation is used, thus expressing the incident and scattered field as:

$$
\overrightarrow{E^{i}}(\rho, \phi)=\sum_{p=-N_{i}}^{N_{1}} i_{p} J_{p}(K \rho) e^{j p \phi} \tilde{z} \quad \overrightarrow{E^{s}}(\rho, \phi)=\sum_{q=-N_{d}}^{N_{d}} c_{q} H_{q}^{(2)}(K \rho) e^{j \xi \phi} \bar{z}
$$

0-7803-5639-X/99/\$10.00 @1999 IEEE. 


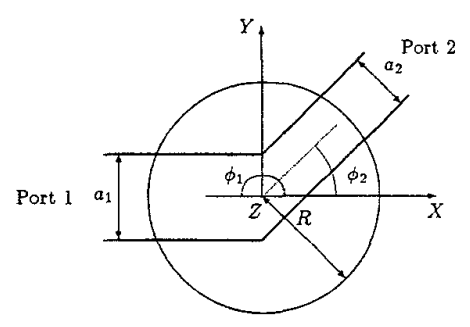

Fig. 1. General layout of the inductive problem

where $i_{p}$ are the incident spectrum and coefficients $c_{q}$ constitute the scattered spectrum. $N_{d}$ must satisfy $N_{d}>K R, R$ being the radius of a circumference that contains the segment, and must satisfy $N_{d}>K R, R$ being the radius of a circumference that contains the segment, and
$N_{i}$ must be large enough to correctly reconstruct the incident field inside the circumference that contains the segment. It is convenient for notation to extend both series to the same that contains the segment. It is con

number of terms $N=\max \left(N_{i}, N_{d}\right)$.
Following [9], the 2D multiple scattering problem is solved by obtaining a joint transfer function for each element of the inductive problem, which relates the spectra of the incident and scattered electric fields in the following way:

$$
\left[c_{q}\right]=\underline{\underline{D}} \cdot\left[i_{p}\right] \rightarrow c_{q}=\sum_{p=-N_{i}}^{N_{i}} D_{q p} i_{p}
$$

Next, to obtain the multimode scattering matrix of the problem a mode-matching technique must be applied. To proceed in this way, the transversal fields at the input ( $i=1)$ and output $(i=2)$ ports must be written as:

$$
\begin{aligned}
& \vec{E}_{t}^{i}(\rho, \phi)=\sum_{m=1}^{M_{i}} a_{m}^{i} e_{i m}^{\prime \prime}\left(x_{i}\right) e^{-\gamma_{m}^{i} z_{i}} \hat{z}+\sum_{m=1}^{M_{i}} b_{m}^{i} e_{i m}^{\prime \prime}\left(x_{i}\right) e^{\gamma_{m}^{i} z_{i}} \hat{z} \\
& \vec{H}_{i}^{i}(\rho, \phi)=\sum_{m=1}^{M_{i}}-a_{m}^{i} Y_{T E_{m 0}}^{i} e_{i m}^{\prime \prime}\left(x_{i}\right) e^{-\gamma_{m}^{i} z_{i}} \hat{x}_{i}+\sum_{m=1}^{M_{i}} b_{m}^{i} Y_{T E_{m}}^{i} e_{i m}^{\prime \prime}\left(x_{i}\right) e^{\gamma_{m}^{i} z_{i}} \hat{x}_{i}
\end{aligned}
$$

where $M_{i}$ is the number of $T E_{m 0}$ modes in the $i$-th port, and

$$
\begin{aligned}
& x_{i}=\rho \sin \left(\phi_{i}-\phi\right)+\frac{a_{i}}{2}, \quad \mathrm{i}=1,2 \\
& z_{i}=-\rho \cos \left(\phi-\phi_{i}\right), \quad \mathrm{i}=1,2 \\
& \hat{x}_{i}=\hat{z} \times \hat{z}_{i}=-\hat{\phi}\left(\hat{\phi}_{i}\right)=\sin \phi_{1} \hat{x}-\cos \phi_{i} \hat{y} \quad, \quad i=1,2 \\
& e_{i m}^{\prime \prime}\left(x_{i}\right)=\left\{\begin{array}{cc}
-\frac{2}{\sqrt{a_{i} b_{i}}} \sin \left(\frac{m \pi}{a_{i}} x_{i}\right) & \left|\phi-\phi_{i}\right| \leq \phi_{i M} \\
0 & \left|\phi-\phi_{i}\right|>\phi_{i M}
\end{array}, \quad i=1,2, \quad[3]\right. \\
& \phi_{i M}=\arcsin \left(\frac{a_{i}}{2 \rho}\right), i=1,2
\end{aligned}
$$

where $b_{i}$ is the height of the input (output) waveguide.

The boundary conditions for the electric and magnetic fields at the circumference of radius $R$ must be enforced. Thus:

$$
\begin{aligned}
\vec{E}_{t}^{i}(\rho=R, \phi)+\vec{E}_{t}^{s}(\rho=R, \phi) & =\vec{E}_{t}^{1}(\rho=R, \phi)+\vec{E}_{t}^{2}(\rho=\dot{R}, \phi) \\
\vec{H}_{t}^{i}(\rho=R, \phi)+\vec{H}_{t}^{s}(\rho=R, \phi) & =\vec{H}_{t}^{1}(\rho=R, \phi)+\vec{H}_{t}^{2}(\rho=R, \phi)
\end{aligned}
$$


After some mathematical manipulations:

$$
\begin{aligned}
& \sum_{m=1}^{M_{1}+M_{2}} b_{m}\left[Y_{T E_{m 0} 0} e_{m}^{-}(\phi)-\frac{j}{K \eta R} \frac{\mathrm{d} e_{m}^{-}}{\mathrm{d} \phi}+\frac{j}{\eta} e_{m}^{-}(\phi) \otimes W(\phi)\right] \\
= & \sum_{m=1}^{M_{1}+M_{2}} a_{m}\left[Y_{T E_{m 0}} e_{m}^{+}(\phi)+\frac{j}{K^{\prime} \eta R} \frac{\mathrm{d} e_{m}^{+}}{\mathrm{d} \phi}-\frac{j}{\eta} e_{m}^{+}(\phi) \otimes W(\phi)\right]
\end{aligned}
$$

where:

$$
\begin{aligned}
a_{m} & =\left\{\begin{array}{cl}
a_{m}^{1} & m \in\left[1, M_{1}\right] \\
a_{m-M_{1}}^{2} & m \in\left[M_{1}+1, M_{1}+M_{2}\right]
\end{array}\right. \\
b_{m} & =\left\{\begin{array}{cl}
b_{m}^{1} & m \in\left[1, M_{1}\right] \\
b_{m-M_{2}}^{2} & m \in\left[M_{1}+1, M_{1}+M_{2}\right]
\end{array}\right. \\
e_{m_{n}}^{ \pm}(\phi) & =\left\{\begin{array}{cl}
e_{1 m}^{\prime \prime}\left(x_{1}(R, \phi)\right) e^{\mp \gamma_{m}^{2} z_{2}(R, \phi)} & m \in\left[1, M_{1}\right] \\
e_{2 m-M_{1}}^{\prime \prime}\left(x_{2}(R, \phi)\right) e^{F r_{m}^{2}-M_{2} z_{2}\left(R_{,}\right)} & m \in\left[M_{1}+1, M_{1}+\right.
\end{array}\right. \\
W(\phi) & =\sum_{p=-\infty}^{\infty}\left[\frac{J_{p}^{\prime}(K R)+\sum_{q=-N}^{N} D_{q p} H_{q}^{(2)^{\prime}}(K R) e^{j(q-p) \phi}}{J_{p}(K R)+\sum_{q=-N}^{N} D_{q p} H_{q}^{(2)}(K R) e^{j(q-p) \phi}}\right] e^{j p \phi}
\end{aligned}
$$

Projecting expression (12) to $e_{n}^{-}(\phi)$ and repeating such operation for $n=1$ to $M_{1}+M_{2}$, the following matrix equation is obtained:

where the elements of $\triangleq$ and $\underline{\underline{Q}}$ are

$$
\begin{aligned}
\Delta_{m n} & =\int_{0}^{2 \pi}\left[Y_{T E_{n 0}} e_{n}^{-}(\phi)-\frac{j}{K \eta R} \frac{\mathrm{d} e_{n}^{-}}{\mathrm{d} \phi}+\frac{j}{\eta} e_{n}^{-}(\phi) \otimes W(\phi)\right] e_{m}^{-}(\phi)^{*} \mathrm{~d} \phi \\
Q_{m n} & =\int_{0}^{2 \pi}\left[Y_{T E_{n 0}} e_{n}^{+}(\phi)+\frac{j}{K \eta R} \frac{\mathrm{d} e_{n}^{+}}{\mathrm{d} \phi}-\frac{j}{\eta} e_{n}^{+}(\phi) \otimes W(\phi)\right] e_{m}^{-}(\phi)^{*} \mathrm{~d} \phi
\end{aligned}
$$

Finally, the multimode scattering matrix for the inductive problem is easily obtained as:

$$
\underline{b}=\underline{\underline{\Delta}} \underline{\underline{Q}}^{-1} \underline{\underline{a}}=\underline{\underline{S}} \underline{a}
$$

III. RESULTS

The new hybrid mode-matching method has been used to calculate some classical inductive problems in order to test its accuracy. Figure $2 \mathrm{a}$ shows the circuital parameters of a waveguide with an inductive circular metallic post. The same results for an off-centred post $\left(x_{0}=0.3 a\right)$ are depicted in figure $2 \mathrm{~b}$. In both cases the mode-matching results are in good agreement with the literature [7], and the computational time required for one frequency agreement with the literature [7], and the computational time required for one frequency
point with mode-matching was around 1 second with Pentium $166 \mathrm{MHz}$. The circuital papoint with mode-matching was around 1 second with Pentium $166 \mathrm{MHz}$. The circuital pa-
rameters of an inductive iris and an inductive aperture are shown in figure $2 \mathrm{c}$, also providing rameters of an inductive iris and an inductive aperture are shown in figure $2 c$, also providing
good agreement with the literature [3]. Finally, the scattering parameter of a dielectric cirgood agreement with the literature [3]. Finally, the scattering parameter of a dielectric cir-
cular post is illustrated in figure $2 d$ and compared with $[10](\alpha=0,714 \lambda, d=0.1 a)$. The time required for each frequency point with mode-matching was around 2 seconds.

$$
\text { IV. Conclusions }
$$

A new hybrid mode-matching method for characterizing inductive problem in waveguiding structures has been presented. The method makes use of an open-space spectral formulation for the efficient solution of the inductive problem, and a mode-matching approach for obtaining the multimode scattering matrix of the problem. The complete method is presented and applied to the analysis of several inductive discontinuities and obstacle posts placed in a rectangular waveguide. A comparison of these results with the ones from the technical literature is also discussed, thus showing the good accuracy of the results obtained with the new method derived. 


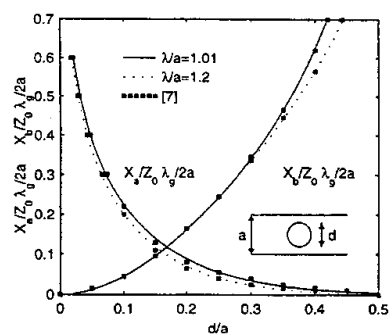

(a)

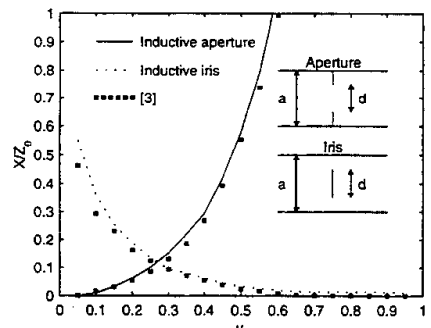

(c)

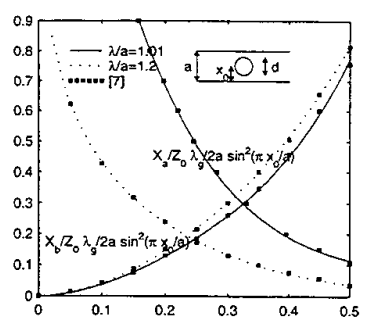

(b)

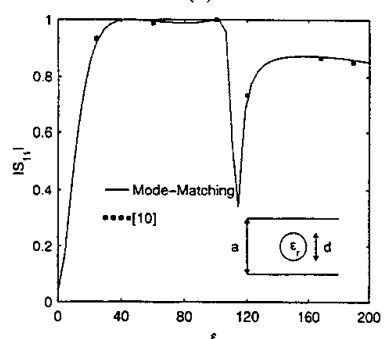

(d)

Fig. 2. Comparison with literature for some classical inductive problems. (a) Centred metallic circular post. (b) Off-centred metallic circular post. (c) Centred dielectric circular post. (d) Inductive iris and

REFERENCES

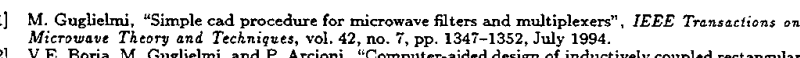
v.E. Boria, M. Guglelms, and $P$. Arcion," "Computer-aided design of inductively coupled rect angular Aaveguide

3] N. Mareuvitz, Waveguide Handbook, IEE Electromagnetic Wave Series $21,1986$.
4. Itoh, Numerical Technigues for Microwave and Millimeter-Wave Passive Structures, John Wiley F. Aons, New York, 1989,

tinuity problens. Collucci, and R. Sorrentino, "Admittance matrix formulation of waveguide disconFicrowave Theory ad M. Guglielmi, G. Gheri, M. Calamia, and G. Pelosi, "Rigurous mutimode network numerical repre-
sentation of inductive step", IEEE Transactions on Microwave Theory and Techniques, vol. 42, no. 2 , Leviatan. Y., P.G. Li, A.X. Adams, and Perinj J., "Single-post inductive obstacle in rectangular waveguide", IEEE Transactions on Microwave Theory and Technigues, vol. 31, no. 10, pp. 806-811, October 1983 .

(8) A. Boetteger. Th. Sieverding, P. Krauss, and F. Arndt, "Fast boundary contour mode-matching method Cor the cad of circular post coupled resonator filters", in Proceedings of the 28th European Microwave Conference, October 1998, pp. $712-715$.

and M. Ferrando, "Generalised iterative method for solving $2 d$ techniques", IEE Proceedings on Microwave, Antennas and [10] Eropagation, vol. 144, no. 2, pp. 73-80, 1997.
Transactions on Microweve Theory and Techniques, vol. MTT-17, no. 3, pp. 148-153, 1969. 